\title{
Hormonal contraceptive use and prevalence of premenstrual symptoms in a multiethnic Canadian population
}

\author{
Alicia Caroline Jarosz, Joseph Jamnik and Ahmed El-Sohemy ${ }^{*}$ (D)
}

\begin{abstract}
Background: Hormonal contraceptive use may be associated with a reduction in some premenstrual symptoms, however, the evidence remains equivocal. The objectives of the present study were to investigate the associations between ethnicity and hormonal contraceptive use with premenstrual symptoms.

Methods: One thousand one hundred two women participating in the Toronto Nutrigenomics and Health Study provided data on their premenstrual symptoms and hormonal contraceptive use. Severity of symptoms was classified as none, mild, moderate, or severe. Prevalence of premenstrual symptoms was determined in the total population and among major ethnic groups. Logistic regressions were used to determine the association between ethnicity and prevalence of premenstrual symptoms. Logistic regressions were used to determine the associations between hormonal contraceptive use, and premenstrual symptoms, adjusting for ethnicity and other covariates.

Results: Prevalence of individual symptoms varied, and the most commonly reported were cramps (75\%), bloating (75\%), mood swings (73\%), increased appetite (64\%), and acne (62\%). Prevalence of cramps differed between ethnic groups with East Asians reporting a lower prevalence than Caucasians and South Asians $(p<0.05)$. Use of hormonal contraceptives was associated with a lower RR ( $95 \% \mathrm{Cl}$ ) of experiencing moderate/severe: cramps (0.82, 0. 72-0.93), clumsiness $(0.22,0.07-0.73)$, confusion $(0.22,0.09-0.54)$ and desire to be alone $(0.45,0.28-0.73)$. Hormonal contraceptive use was not associated with the risk of premenstrual symptoms at mild severity. Hormonal contraceptive use was not associated with symptoms of anxiety, bloating, mood swings, increased appetite, acne, fatigue, sexual desire, depression, nausea, headache and insomnia.
\end{abstract}

Conclusion: This study demonstrates that East Asians may be at a lower risk of experiencing premenstrual cramps and that hormonal contraceptive use is associated with a lower risk of experiencing many, but not all, premenstrual symptoms at moderate/severe severity.

Keywords: Premenstrual symptoms, Prevalence, Ethnicity, Hormonal contraceptives

\section{Background}

Premenstrual symptoms include a wide range of physical, psychological and behavioral symptoms, which occur in the late luteal phase of a woman's reproductive cycle and subside a few days following the onset of menses [1]. Many symptoms have been described to date, and a few most commonly experienced somatic symptoms are bloating, headache, fatigue, and muscle cramps.

\footnotetext{
* Correspondence: a.el.sohemy@utoronto.ca

Department of Nutritional Sciences, University of Toronto, Room 350, 150 College St, Toronto, ON M5S 3E2, Canada
}

Behavioural and psychological symptoms are also commonly experienced, such as anxiety, mood swings, changes in appetite, and depression [2,3]. It is estimated that more than $80 \%$ of women regularly experience premenstrual symptoms, however, prevalence varies between studies and populations [4-10]. It is generally accepted that the prevalence is influenced by factors such as body weight and age, however, the association with ethnicity has been inconsistent $[11,12]$.

Little is known about the pathophysiology of premenstrual symptoms, and consequently, few effective therapies 
have been developed for them [1]. Due to the timing of the symptoms, changes in plasma levels of progesterone and estradiol are thought to be involved in their etiology [1]. Stabilizing fluctuations of these hormones during the luteal phase with the use of hormonal contraceptives may be effective in treating premenstrual symptoms [1], but the evidence has been inconsistent $[1,2]$.

Due to the large variation in frequencies of reported symptoms and their possible associations with ethnicity and hormonal contraceptive use, the objectives of this study were to determine the prevalence of various premenstrual symptoms in a multiethnic Canadian population and to assess their associations with hormonal contraceptive use.

\section{Methods}

\section{Study sample}

Subjects included 1636 men and women aged 2029 years who participated in the Toronto Nutrigenomics and Health (TNH) study, which is a cross-sectional examination of young adults investigating genetics, lifestyle, and biomarkers of health [13, 14]. Recruitment occurred between 2004 and 2010. Participants provided overnight fasting blood samples and completed a general health and lifestyle questionnaire (GHLQ) which included questions regarding premenstrual symptoms, hormonal contraceptive (HC) use, and physical activity. Exclusion criteria included current pregnancy or breastfeeding. The study protocol was approved by the Ethics Review Board of the University of Toronto and participants provided written informed consent.

From the initial 1636 subjects, 520 men were excluded, 10 subjects were excluded due to missing GHLQ information, and 4 were excluded for lack of blood samples. The remaining 1102 female subjects were categorized into four ethnic groups based on self-reported ethnic status: Caucasian $(n=514)$, East Asian $(n=401)$, South Asian $(n=105)$, or Other $(n=82)$, as described previously [15]. Caucasians included those self-reported as European, Middle Eastern, or Hispanic. East Asians consisted of Chinese, Japanese, Korean, Filipino, Vietnamese, Thai, and Cambodian. South Asians included Bangladeshi, Indian, Pakistani, and Sri Lankan. The Other category included self-reported ethnicities of Aboriginal Canadians, Afro-Caribbeans, and those who selfreported belonging to $\geq 2$ ethnic groups not included in the same category.

\section{GHLQ}

Hormonal contraceptive use was self-reported in the GHLQ. Subjects were categorized as HC users $(n=320)$ and non-users $(n=782)$. HC users included subjects indicating current use of HCs, regardless of $\mathrm{HC}$ type or delivery method (transdermal, oral, vaginal, injection, etc.). Subjects also reported use of any medications in the past month. Use of anti-depressants, analgesics, or anxiolytics was considered in the present study as 'PMS medication use, due to their effects on premenstrual symptoms.

\section{Anthropometrics and physical activity}

Subjects' height and weight were measured using the protocol previously described by Garcia-Bailo et al. (2012) [16]. Subjects wore light clothing and removed their shoes during the measurements. Body mass index (BMI) was subsequently calculated in $\mathrm{kg} / \mathrm{m}^{2}$. Subjects self-reported their physical activity in the GHLQ by estimating the amount of time they spent sleeping and engaging in light, moderate, and vigorous activity. Values were then converted into metabolic equivalent (MET) levels.

\section{Plasma samples and vitamin D measurement}

Participants provided blood samples following a minimum 12-h overnight fast. Participants experiencing a temporary inflammatory condition (including a recent piercing or tattoo, acupuncture, a medical or dental procedure, a vaccination or immunization, flu, an infection, or a fever) underwent a two-week recovery period prior to providing blood samples. Samples were collected at LifeLabs Medical Laboratory Services (Toronto, Ontario, Canada), and 25-hydroxyvitamin D $(25(\mathrm{OH}) \mathrm{D})$ levels were measured at the University Health Network Specialty Lab at Toronto General Hospital (Toronto, Ont., Canada). Plasma 25(OH)D was measured by high-performance liquid chromatography-tandem mass spectrometry.

\section{Premenstrual symptoms}

Premenstrual symptoms and severities were self-reported in a questionnaire included in the GHLQ. The questionnaire included the following symptoms: cramps; bloating/ swelling/breast tenderness; mood swings/irritability/angry outbursts; increased appetite/food cravings; acne; sexual desire/activity change; fatigue; anxiety/tension/nervousness; depression; desire to be alone; confusion/difficulty concentrating/forgetfulness; nausea; insomnia; headache; and clumsiness. Symptom severities were classified as none, mild, moderate, or severe. Subjects were asked to indicate the severity at which they experienced each symptom, within the 5 days before the onset of their period and ending by the 4th day of their period. Subjects could also list other premenstrual symptoms experienced, however, due to the scarcity of other symptoms they were not included in the analyses. The premenstrual questionnaire was self-developed for the TNH study based on commonly reported symptoms and previously validated questionnaires. 


\section{Statistical analysis}

All statistical analyses were conducted using SAS (version 9.4; SAS Institute Inc., Cary, NC, USA). The $\alpha$ was set at 0.05 and all reported $p$-values are 2 -sided. Subject characteristics were compared between $\mathrm{HC}$ users and non-users by chi-square analysis for categorical variables and t-tests for continuous variables. Distribution of continuous variables was assessed prior to analysis and log-transformed BMI was used in all subsequent analyses. Crude mean BMI values were reported for ease of interpretation.

The prevalence of premenstrual symptoms was defined as the frequency of subjects experiencing the symptoms at any severity (mild, moderate, or severe). Prevalence was calculated for each symptom in the total population, and separately for the major ethnic groups: Caucasians $(n=514)$, East Asians $(n=401)$, South Asians $(n=105)$, and Other $(n=82)$. Logistic regressions were used to determine differences in the prevalence of symptoms between the four ethnic groups. $P$ values were calculated in both unadjusted models as well as adjusted models which included the following covariates: age, BMI, HC use, physical activity, PMS medication use and plasma 25(OH)D concentrations. BenjaminiYekutieli adjustments for multiple comparisons were applied (15 tests, $\alpha=0.05: p<0.015)$. Differences in the prevalence of each symptom between each pair of ethnic groups were also examined (Caucasians vs East Asians; Caucasians vs South Asians; Caucasians vs Other; East Asians vs South Asians; East Asians vs Other; South Asians vs Other) using logistic regressions.

Logistic regressions were used to examine the associations between $\mathrm{HC}$ use and premenstrual symptom severities. The proc. genmod procedure was conducted with a binomial distribution and a log link function. Moderate and severe symptom severities were combined due to the small number of subjects reporting severe symptoms. Relative risks (RR) and 95\% confidence intervals (CI) were reported for associations between $\mathrm{HC}$ use and premenstrual symptoms. Univariate models were first used in Model 1, followed by multivariate models in Model 2 which adjusted for ethnicity, BMI, physical activity, PMS medication use and age. Covariates were selected based on their associations with $\mathrm{HC}$ use or premenstrual symptoms in the TNH study population and previous studies. Benjamini-Yekutieli adjustments for multiple comparisons were applied (30 tests, $\alpha=0.05: p<0.013$ ).

\section{Results}

\section{Study sample}

Subject characteristics are shown in Table 1. The mean age of participants was 22.6 years. HC users were on average older (23 years) than non-users (22.4 years) $(p=0.0006)$. HC use differed between ethnic groups, with Caucasian women reporting the greatest use of
Table 1 Subject characteristics stratified by Hormonal Contraceptive $(\mathrm{HC})$ use

\begin{tabular}{llll}
\hline & HC non-users (\%) & HC users (\%) & p-value \\
\hline $\mathrm{N}$ & 782 & 320 & 0.0006 \\
Age (years) & $22.4 \pm 0.1$ & $23.0 \pm 0.1$ & \\
Ethnicity (\%) & & & $<0.0001$ \\
$\quad$ Caucasian & $293(57)$ & $221(43)$ & \\
$\quad$ East Asian & $348(87)$ & $53(13)$ & \\
$\quad$ South Asian & $87(83)$ & $18(17)$ & \\
$\quad$ Other & $54(66)$ & $28(34)$ & 0.11 \\
Body mass index (kg/m $\left.{ }^{2}\right)^{*}$ & $22.4 \pm 0.1$ & $22.7 \pm 0.2$ & 0.007 \\
Physical activity (met-h/wk) & $7.5 \pm 0.1$ & $8.1 \pm 0.2$ & 0.08 \\
PMS Medication Use & $205(26)$ & $68(21)$ & \\
\hline Shown are crude means and standard errors continuous vables and
\end{tabular}

Shown are crude means and standard errors of continuous variables, and $\mathrm{n}$ (\%) of categorical variables

$p$-values were obtained using chi-square tests for categorical variables and ttests for continuous variables

*indicates log-transformed variable was used for obtaining $p$-value

HCs (43\%), followed by Other (34\%), South Asians (17\%) and East Asians (13\%). Reported physical activity was greater for $\mathrm{HC}$ users (8.1 met-hours/week) than non-users (7.5 met-hours/week $)(p=0.007)$. Logtransformed BMI and PMS medication use did not differ between $\mathrm{HC}$ users and non-users.

\section{Prevalence of premenstrual symptoms}

Prevalence of experiencing any premenstrual symptoms in the total population was $99 \%$, and did not differ significantly between ethnic groups $(p=0.11)$. Prevalence of each premenstrual symptom in the total population and stratified by ethnicity is shown in Table 2 . The most common symptoms experienced were cramps (75\%), bloating (75\%), mood swings (73\%), increased appetite (64\%), and acne (62\%). Other premenstrual symptoms experienced were fatigue (55\%), sexual desire (50\%), anxiety (37\%), desire to be alone (33\%), depression (29\%), headache $(27 \%)$, confusion (21\%), clumsiness (15\%), nausea $(15 \%)$, and insomnia (11\%).

In the unadjusted model symptom prevalence differed between ethnic groups for symptoms of cramps, bloating, sexual desire, headache, and confusion $(p<0.05)$. However, after adjustments for age, BMI, HC use, physical activity, medication use, and plasma $25(\mathrm{OH}) \mathrm{D}$ concentrations, only the prevalence of cramps differed between ethnic groups $(p<0.05)$. This association met adjustments for multiple comparisons $(p<0.015)$, where East Asians reported a lower prevalence of cramps than Caucasians and South Asians. Prevalence of bloating, mood swings, increased appetite, acne, fatigue, sexual desire, anxiety, desire to be alone, depression, headache, confusion, clumsiness, nausea, and insomnia did not differ between ethnic groups in the adjusted model. 
Table 2 Premenstrual symptom prevalence by ethnicity

\begin{tabular}{|c|c|c|c|c|c|c|c|}
\hline \multirow[t]{2}{*}{ Symptom } & \multirow{2}{*}{$\begin{array}{l}\text { Total (\%) } \\
N=1102\end{array}$} & \multirow{2}{*}{$\begin{array}{l}\text { Caucasian (\%) } \\
N=514\end{array}$} & \multirow{2}{*}{$\begin{array}{l}\text { East Asian (\%) } \\
N=401\end{array}$} & \multirow{2}{*}{$\begin{array}{l}\text { South Asian (\%) } \\
N=105\end{array}$} & \multirow{2}{*}{$\begin{array}{l}\text { Other (\%) } \\
N=82\end{array}$} & \multirow{2}{*}{$\begin{array}{l}\text { Model } 1 \\
p \text {-value }\end{array}$} & \multirow{2}{*}{$\begin{array}{l}\text { Model } 2 \\
p \text {-value }\end{array}$} \\
\hline & & & & & & & \\
\hline Cramps & 75 & $79^{\mathrm{a}}$ & $67^{b}$ & $84^{a}$ & $78^{\mathrm{ab}}$ & $<0.0001$ & $<0.0001$ \\
\hline Bloating/Swelling/Breast Tenderness & 75 & 79 & 70 & 70 & 78 & 0.01 & 0.16 \\
\hline Mood Swings//rritability & 73 & 73 & 72 & 74 & 67 & 0.68 & 0.68 \\
\hline Increased Appetite/Food Cravings & 64 & 65 & 63 & 64 & 62 & 0.90 & 0.86 \\
\hline Acne & 62 & 66 & 60 & 52 & 57 & 0.05 & 0.18 \\
\hline Fatigue & 55 & 52 & 56 & 58 & 65 & 0.15 & 0.13 \\
\hline Sexual Desire/Activity Change & 50 & 55 & 42 & 48 & 56 & 0.001 & 0.11 \\
\hline Anxiety/Tension/Nervousness & 37 & 36 & 38 & 34 & 34 & 0.85 & 0.87 \\
\hline Desire to be alone & 33 & 30 & 33 & 42 & 39 & 0.06 & 0.23 \\
\hline Depression & 29 & 30 & 27 & 33 & 28 & 0.57 & 0.63 \\
\hline Headache & 27 & 27 & 23 & 37 & 29 & 0.03 & 0.15 \\
\hline Confusion/Difficulty Concentrating/Forgetfulness & 21 & 18 & 26 & 24 & 18 & 0.02 & 0.17 \\
\hline Clumsiness & 15 & 14 & 18 & 15 & 11 & 0.26 & 0.27 \\
\hline Nausea & 15 & 16 & 11 & 20 & 17 & 0.05 & 0.21 \\
\hline Insomnia & 11 & 9 & 11 & 16 & 13 & 0.16 & 0.63 \\
\hline
\end{tabular}

Sorted by total premenstrual symptom prevalence. Letters indicate prevalence values which differed significantly from each other in the adjusted model $(p<0.05)$

\section{Premenstrual symptom associations with $\mathrm{HC}$ use}

Associations between premenstrual symptoms and $\mathrm{HC}$ use are shown in Table 3. In the unadjusted model, $\mathrm{HC}$ use was associated with a lower risk of experiencing mild acne and the following symptoms at moderate/severe severity: cramps, fatigue, anxiety, clumsiness, confusion, depression, and desire to be alone. In Model 2, which adjusted for ethnicity, BMI, physical activity, PMS medication use and age, $\mathrm{HC}$ use was not associated with any symptoms at mild severity. $\mathrm{HC}$ use was associated with a lower RR (95\% CI) of experiencing moderate/severe: cramps $(0.82,0.72-0.93)$, anxiety $(0.63,0.42-0.95)$, clumsiness $(0.22,0.07-0.73)$, confusion $(0.22,0.09-0.54)$, depression $(0.55,0.34-0.90)$, and desire to be alone $(0.45$, 0.28-0.73). Symptoms of cramps, clumsiness, confusion, and desire to be alone met Benjamini-Yekutieli criteria for multiple comparisons (30 tests, $\alpha=0.05$ : $p<0.013$ ). Premenstrual symptoms of bloating, mood swings, increased appetite, acne, fatigue, sexual desire, headache, nausea, and insomnia were not associated with $\mathrm{HC}$ use in Model 2. Low sample size precluded calculations of adjusted relative risks for moderate/severe nausea, where unadjusted RRs were: $0.53(0.25,1.13)$.

\section{Discussion}

In this study, we investigated the prevalence of 15 common premenstrual symptoms and their associations with hormonal contraceptive use in a multiethnic population of young adults living in Canada. Our findings show that the prevalence of individual premenstrual symptoms varies widely between the symptoms, and we observed ethnic differences in the prevalence of cramps. We also found that $\mathrm{HC}$ use was associated with a lower risk of experiencing several, but not all, premenstrual symptoms at moderate/severe severity. No associations were observed between $\mathrm{HC}$ use and the risk of experiencing mild premenstrual symptoms.

In our population $99 \%$ of the subjects reported experiencing premenstrual symptoms. The same prevalence estimates were found in female university students in Thailand and Iran $[8,9]$. Prevalence reported in other studies have been slightly lower and have ranged from $80 \%$ to $95 \%[4-7,17]$. These variations in prevalence estimates may be explained by differences in symptom assessment, subject population, and subject characteristics such as age [18]. For example, the lowest prevalence of $80 \%$ was reported in a German community survey which included adolescent subjects aged 14-24 years [6]. The inclusion of adolescents could explain the lower prevalence, as was shown in a previous study which found that subjects under 20 or over 45 years of age had the lowest symptom prevalence, with prevalence peaking at age 35 [10]. Alternatively, a survey of only married Iranian women from health clinics aged 20-45 reported a prevalence of $86 \%$ [17]. Two previous studies that included women of similar age as in the present study reported similar prevalence for the various premenstrual symptoms $[8,9]$.

The most commonly experienced symptoms in the present study were cramps (75\%), bloating (75\%), irritability (73\%), increased appetite (64\%), and acne (62\%). These differed from those reported in other studies, and 
Table 3 Associations between $\mathrm{HC}$ use and premenstrual symptom severity

\begin{tabular}{|c|c|c|c|c|c|c|c|}
\hline \multirow[t]{2}{*}{ Symptom } & \multirow[t]{2}{*}{ Severity } & $\mathrm{HC}$ non-users (\%) & HC Users (\%) & Model 1 & Model 1 & Model 2 & Model 2 \\
\hline & & $N=782$ & $N=320$ & Relative Risk & $p$-value & Relative Risk & $p$-value \\
\hline \multirow[t]{3}{*}{ Acne / Skin Blemish } & None & $310(40)$ & $111(35)$ & REF & & REF & \\
\hline & Mild & $318(41)$ & $161(50)$ & $1.17(1.03,1.33)$ & 0.02 & $1.12(0.98,1.28)$ & 0.10 \\
\hline & Moderate/Severe & $154(20)$ & $48(15)$ & $0.91(0.69,1.19)$ & 0.49 & $0.84(0.63,1.12)$ & 0.23 \\
\hline \multirow[t]{3}{*}{ Bloating / Swelling / Breast Tenderness } & None & $207(26)$ & $71(22)$ & REF & & REF & \\
\hline & Mild & $309(40)$ & $143(45)$ & $1.12(0.99,1.26)$ & 0.07 & $1.07(0.95,1.22)$ & 0.27 \\
\hline & Moderate/Severe & $266(34)$ & $106(33)$ & $1.06(0.92,1.23)$ & 0.39 & $1.00(0.86,1.16)$ & 0.99 \\
\hline \multirow[t]{3}{*}{ Cramps } & None & $185(24)$ & $89(28)$ & REF & & REF & \\
\hline & Mild & $256(33)$ & $128(40)$ & $1.02(0.89,1.16)$ & 0.82 & $0.93(0.81,1.07)$ & 0.29 \\
\hline & Moderate/Severe & $341(44)$ & $103(32)$ & $0.83(0.72,0.96)$ & 0.01 & $0.82(0.71,0.93)$ & 0.002 \\
\hline \multirow{3}{*}{$\begin{array}{l}\text { Mood Swings / Crying Easily / Irritability / } \\
\text { Angry Outbursts }\end{array}$} & None & $212(27)$ & $92(29)$ & REF & & REF & \\
\hline & Mild & $287(37)$ & $128(40)$ & $1.01(0.88,1.16)$ & 0.87 & $1.02(0.88,1.18)$ & 0.80 \\
\hline & Moderate/Severe & $283(36)$ & $100(31)$ & $0.91(0.78,1.06)$ & 0.24 & $0.89(0.76,1.06)$ & 0.19 \\
\hline \multirow[t]{3}{*}{ Increased Appetite / Food Cravings } & None & $282(36)$ & $112(35)$ & REF & & REF & \\
\hline & Mild & $232(30)$ & 107 (33) & $1.08(0.91,1.28)$ & 0.35 & $1.06(0.89,1.27)$ & 0.49 \\
\hline & Moderate/Severe & $268(34)$ & $101(32)$ & $0.97(0.82,1.15)$ & 0.75 & $0.97(0.82,1.16)$ & 0.76 \\
\hline \multirow[t]{3}{*}{ Fatigue } & None & $342(44)$ & $153(48)$ & REF & & REF & \\
\hline & Mild & $245(31)$ & 107 (33) & $0.99(0.83,1.17)$ & 0.87 & $1.00(0.83,1.20)$ & 0.97 \\
\hline & Moderate/Severe & $195(25)$ & $60(19)$ & $0.78(0.61,0.99)$ & 0.04 & $0.82(0.64,1.05)$ & 0.11 \\
\hline \multirow[t]{3}{*}{ Headache } & None & $575(74)$ & $231(72)$ & REF & & REF & \\
\hline & Mild & $131(17)$ & $58(18)$ & $1.08(0.82,1.43)$ & 0.58 & $1.11(0.84,1.49)$ & 0.46 \\
\hline & Moderate/Severe & $76(10)$ & $31(10)$ & $1.01(0.68,1.50)$ & 0.95 & $1.01(0.67,1.51)$ & 0.97 \\
\hline \multirow[t]{3}{*}{ Anxiety / Tension / Nervousness } & None & $480(61)$ & $220(69)$ & REF & & REF & \\
\hline & Mild & $201(26)$ & $73(23)$ & $0.84(0.67,1.06)$ & 0.15 & $0.88(0.69,1.13)$ & 0.31 \\
\hline & $\begin{array}{l}\text { Moderate/ } \\
\text { Severe }\end{array}$ & $101(13)$ & $27(8)$ & $0.63(0.42,0.94)$ & 0.02 & $0.63(0.42,0.95)$ & 0.03 \\
\hline \multirow[t]{3}{*}{ Clumsiness } & None & $655(84)$ & $278(87)$ & REF & & REF & \\
\hline & Mild & $90(12)$ & $39(12)$ & $1.02(0.72,1.45)$ & 0.92 & $1.07(0.74,1.56)$ & 0.71 \\
\hline & Moderate/Severe & $37(5)$ & $3(1)$ & $0.20(0.06,0.64)$ & 0.007 & $0.22(0.07,0.73)$ & 0.01 \\
\hline \multirow{3}{*}{$\begin{array}{l}\text { Confusion / Difficulty Concentrating / } \\
\text { Forgetfulness }\end{array}$} & None & $599(77)$ & $267(83)$ & REF & & REF & \\
\hline & Mild & $121(15)$ & $48(15)$ & $0.91(0.67,1.23)$ & 0.53 & $1.00(0.72,1.38)$ & 1.00 \\
\hline & Moderate/Severe & $62(8)$ & $5(2)$ & $0.20(0.08,0.48)$ & 0.0004 & $0.22(0.09,0.54)$ & 0.001 \\
\hline \multirow[t]{3}{*}{ Sexual Desire / Activity Change } & None & $407(52)$ & $147(46)$ & REF & & REF & \\
\hline & Mild & $233(30)$ & $112(35)$ & $1.19(1.00,1.41)$ & 0.05 & $1.14(0.95,1.37)$ & 0.16 \\
\hline & Moderate/Severe & $142(18)$ & $61(19)$ & $1.13(0.88,1.46)$ & 0.33 & $0.96(0.74,1.23)$ & 0.74 \\
\hline \multirow[t]{3}{*}{ Insomnia } & None & $688(88)$ & $293(92)$ & REF & & REF & \\
\hline & Mild & $75(10)$ & $25(8)$ & $0.80(0.52,1.23)$ & 0.31 & $0.91(0.57,1.43)$ & 0.68 \\
\hline & Moderate/Severe & $19(2)$ & $2(1)$ & $0.25(0.06,1.08)$ & 0.06 & $0.23(0.05,0.99)$ & 0.05 \\
\hline \multirow[t]{3}{*}{ Nausea $^{a}$} & None & $664(85)$ & $277(87)$ & REF & & REF & \\
\hline & Mild & $81(10)$ & $35(10)$ & $1.03(0.71,1.50)$ & 0.87 & $0.95(0.64,1.41)$ & 0.80 \\
\hline & Moderate/Severe & $37(5)$ & $8(3)$ & $0.53(0.25,1.13)$ & 0.10 & N/A & N/A \\
\hline \multirow[t]{3}{*}{ Depression } & None & $543(69)$ & $236(74)$ & REF & & REF & \\
\hline & Mild & $150(19)$ & $65(20)$ & $1.00(0.77,1.29)$ & 0.99 & $0.96(0.73,1.26)$ & 0.77 \\
\hline & Moderate/Severe & $89(11)$ & $19(6)$ & $0.53(0.33,0.85)$ & 0.008 & $0.55(0.34,0.90)$ & 0.02 \\
\hline
\end{tabular}


Table 3 Associations between HC use and premenstrual symptom severity (Continued)

\begin{tabular}{|c|c|c|c|c|c|c|c|}
\hline \multirow[t]{2}{*}{ Symptom } & \multirow[t]{2}{*}{ Severity } & $\mathrm{HC}$ non-users (\%) & HC Users (\%) & Model 1 & Model 1 & Model 2 & Model 2 \\
\hline & & $N=782$ & $N=320$ & Relative Risk & $p$-value & Relative Risk & $p$-value \\
\hline \multirow[t]{3}{*}{ Desire to be alone } & None & $499(64)$ & $238(75)$ & REF & & REF & \\
\hline & Mild & $180(23)$ & $62(19)$ & $0.78(0.60,1.01)$ & 0.06 & $0.80(0.62,1.04)$ & 0.10 \\
\hline & Moderate/Severe & $103(13)$ & $19(6)$ & $0.43(0.27,0.69)$ & 0.0004 & $0.45(0.28,0.73)$ & 0.001 \\
\hline
\end{tabular}

Model 1 contains unadjusted relative risks and $p$-values

Model 2 contains relative risks and $p$-values adjusted for ethnicity, log-transformed BMI, physical activity, age, and medication use

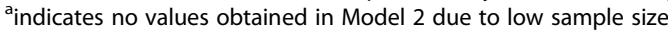

as expected, investigations into the nature of the most commonly experienced symptoms have yielded varying results depending on the population studied $[17,19,20]$. In a recent survey of Iranian women, the most common symptoms reported were tiredness $(70 \%)$, backache (68\%), headache (59\%), and insomnia (50\%) [17]. The most common premenstrual symptoms reported in a population of Turkish medical students were bloating (90\%), irritability (88\%), breast tenderness $(83 \%)$, and anxiety (74\%) [19]. However, a study involving a Mexican population demonstrated abdominal cramping to be the most prevalent symptom (54\%), while only $8 \%$ of women reported irritability [20]. Discrepancies in the prevalence of symptoms may be explained by several factors including variations in premenstrual symptom questionnaires, BMI, age, cultural factors, and environmental exposures. The questionnaire used in the present study differed from those used by others [17, 19, 20], which could account for some of the variation in symptom reporting. For example, the questionnaire used by Goker et al. did not include symptoms of acne, appetite changes, or cramps which were among the five most commonly experienced symptoms in the present population [19].

The effect of ethnicity in relation to premenstrual symptoms remains controversial. Sternfeld et al. showed that relative to Whites, Hispanics reported a greater severity of premenstrual symptoms whereas Asians reported a lesser severity [12]. Several studies involving US populations have shown significant differences in symptom prevalence between White and Black women, but these racial differences were not present for all symptoms [21-23]. This is in line with the results of the present study which revealed ethnic differences in the prevalence of some, but not all, symptoms and no ethnic differences in the total prevalence. In the present study, many symptoms were observed to differ by ethnicity in our unadjusted models but after adjustments for potential confounding variables these differences were no longer significant. Following adjustments, ethnic differences in prevalence were observed only for cramps which met adjustments for multiple comparisons. East Asian participants reported a lower prevalence of cramps compared to Caucasian and South Asian participants. Although this may reflect differences in genetics or cultural factors that may put East Asians at lesser risk of some premenstrual symptoms, it could also be explained by cultural differences in the interpretation and reporting of symptoms [24]. Ethnic differences in premenstrual symptom reporting have been previously observed and it was suggested that differences in the clustering of symptoms in Chinese women compared to Western women may be a result of differences in the conceptualization of the integration of organ systems and their relation to health and disease influenced by Traditional Chinese Medicine [24]. Nonetheless, these findings may guide researchers and healthcare practitioners in determining high-risk populations for premenstrual symptoms, and should be supported by future large-scale studies on Canadian populations.

In the present study, hormonal contraceptive use was associated with a lower risk of experiencing moderate/ severe cramps, desire to be alone, clumsiness and confusion. Use of hormonal contraceptives was not associated with mild premenstrual symptoms. These findings are in agreement with three previous studies that found a decrease in the overall prevalence of symptoms as well as a decrease in the number and severity of emotional symptoms in women using oral contraceptives $[12,25,26]$. Two studies found no association between $\mathrm{HC}$ use and premenstrual symptoms [10, 27]. One study sampling 400 Iranian women observed a greater prevalence of several premenstrual symptoms in $\mathrm{HC}$ users versus nonusers [17]. These studies, however, assessed the effects of $\mathrm{HC}$ use on grouped symptom prevalence and severity, while the present study identified specific premenstrual symptoms and severities which are associated with $\mathrm{HC}$ use. Grouping of symptoms likely accounted for these discrepancies in findings of associations between $\mathrm{HC}$ use and premenstrual symptoms. As shown in the present study, not all symptoms are associated with $\mathrm{HC}$ use and including their prevalence likely impacted previous findings. The present findings emphasize the importance of examining individual premenstrual symptoms in research investigating the efficacy of treatments for premenstrual symptoms.

The observed improvement of premenstrual symptoms with $\mathrm{HC}$ use has largely been attributed to stabilizing ovarian sex steroid fluctuations during the reproductive 
cycle [28]. Treatments preventing ovulation, such as longacting GnRH agonists and bilateral oophorectomy, have been highly effective in diminishing premenstrual symptoms [28]. HCs may present a more favorable option for the management of premenstrual symptoms as they are accompanied by far fewer and less severe side effects [1]. Some HCs also possess anti-aldosterone and antiandrogenic properties that likely enhance their effects on premenstrual symptoms [1, 29]. There is some evidence that the effect of $\mathrm{HC}$ use on premenstrual symptoms is dependent on the HC formulation and regimen [30, 31]. In the present study, sample size limitations precluded the ability to study the effects of different $\mathrm{HC}$ formulations on premenstrual symptoms. Interestingly, $\mathrm{HC}$ use is associated with a higher concentration of pro-inflammatory proteins [32] which have also been linked to an increase in the severity of some premenstrual symptoms [33, 34].

The present study has some limitations. The questionnaire did not specify a retrospective time-frame for experiencing the listed premenstrual symptoms, which may have resulted in an under-reporting or over-reporting of symptoms depending on the participant's interpretation of the question. The present study relied on retrospective symptom reporting which may result in an over-reporting of premenstrual symptoms [35]. This likely did not impact the associations with $\mathrm{HC}$ use, as ethnicity was adjusted for in that analysis and there is no evidence that symptom over-reporting would be more common in $\mathrm{HC}$ users than non-users.

\section{Conclusions}

This cross-sectional examination of a young multiethnic population of Canadian women shows that $99 \%$ of women experience some type of premenstrual symptom, while the prevalence of individual symptoms varies widely. Findings suggest that ethnicity is not a risk factor for experiencing most premenstrual symptoms, although East Asians may be at a lower risk of premenstrual cramps. Use of hormonal contraceptives may put women at a lower risk of experiencing some premenstrual symptoms, but this effect may be dependent on the nature and severity of the symptom.

\section{Abbreviations \\ 25(OH)D: 25-Hydroxyvitamin D; BMI: Body mass index; Cl: Confidence interval; GHLQ: General health and lifestyle questionnaire; HC: Hormonal contraceptive; MET: Metabolic equivalent; RR: Relative risk; TNH: Toronto Nutrigenomics and Health}

\section{Funding}

This work was supported by the Natural Sciences and Engineering Research Council. A.C.J. is a recipient of a Natural Sciences and Engineering Research Council Graduate Scholarship and an Ontario Graduate Scholarship. J.J. is a recipient of a Canadian Institutes of Health Research Doctoral Research Award. The funders had no role in study design, data collection and analysis, decision to publish, or preparation of the manuscript.

\section{Availability of data and materials}

The datasets used and/or analysed during the current study are available from the corresponding author on reasonable request.

\section{Authors' contributions}

$A C J$ and $J J$ performed data analysis. ACJ contributed to manuscript preparation and literature review. ACJ, JJ, and AE-S all contributed to study design, data interpretation, and manuscript revision. All authors have read and approved the final manuscript.

\section{Ethics approval and consent to participate}

The study protocol was approved by the Ethics Review Board of the University of Toronto. It also conforms to standards for the use of human subjects in research as outlined in the Declaration of Helsinki. Written, informed consent was obtained from all participants in the study.

\section{Consent for publication}

Not applicable.

\section{Competing interests}

The authors declare that they have no competing interests.

\section{Publisher's Note}

Springer Nature remains neutral with regard to jurisdictional claims in published maps and institutional affiliations.

Received: 3 August 2017 Accepted: 20 September 2017

Published online: 26 September 2017

\section{References}

1. Rapkin AJ, Akopians AL. Pathophysiology of premenstrual syndrome and premenstrual dysphoric disorder. Menopause Int. 2012;18(2):52-9.

2. Braverman PK. Premenstrual syndrome and premenstrual dysphoric disorder. J Pediatr Adolesc Gynecol. 2007;20(1):3-12.

3. Halbreich $U$. The diagnosis of premenstrual syndromes and premenstrual dysphoric disorder-clinical procedures and research perspectives. Gynecol Endocrinol. 2004;19(6):320-34.

4. Tschudin S, Bertea PC, Zemp E. Prevalence and predictors of premenstrual syndrome and premenstrual dysphoric disorder in a population-based sample. Arch Womens Ment Health. 2010;13(6):485-94.

5. Tolossa FW, Bekele ML. Prevalence, impacts and medical managements of premenstrual syndrome among female students: cross-sectional study in College of Health Sciences, Mekelle University, Mekelle, northern Ethiopia. BMC Womens Health. 2014;14:52.

6. Wittchen HU, Becker E, Lieb R, Krause P. Prevalence, incidence and stability of premenstrual dysphoric disorder in the community. Psychol Med. 2002; 32(1):119-32.

7. Takeda T, Tasaka K, Sakata M, Murata Y. Prevalence of premenstrual syndrome and premenstrual dysphoric disorder in Japanese women. Arch Womens Ment Health. 2006:9(4):209-12.

8. Thu MD, Sawhsarkapaw OG. Premenstrual syndrome among Female University Students in Thailand. AU JT. 2006;9(3):158-62.

9. Bakhshani NM, Mousavi MN, Khodabandeh G. Prevalence and severity of premenstrual symptoms among Iranian female university students. J Pak Med Assoc. 2009;59(4):205-8.

10. Dennerstein L, Lehert $P$, Backstrom TC, Heinemann K. Premenstrual symptoms - severity, duration and typology: an international cross-sectional study. Menopause Int. 2009;15(3):120-6.

11. Adewuya AO, Loto OM, Adewumi TA. Pattern and correlates of premenstrual symptomatology amongst Nigerian University students. J Psychosom Obstet Gynaecol. 2009;30(2):127-32.

12. Sternfeld B, Swindle R, Chawla A, Long S, Kennedy S. Severity of premenstrual symptoms in a health maintenance organization population. Obstet Gynecol. 2002;99(6):1014-24.

13. Ozsungur S, Brenner D, El-Sohemy A. Fourteen well-described caffeine withdrawal symptoms factor into three clusters. Psychopharmacology. 2009; 201(4):541-8.

14. Cahill L, Corey PN, El-Sohemy A. Vitamin C deficiency in a population of young Canadian adults. Am J Epidemiol. 2009;170(4):464-71. 
15. Brenner DR, Boucher BA, Kreiger N, Jenkins D, El-Sohemy A. Dietary patterns in an ethnoculturally diverse population of young Canadian adults. Can J Diet Pract Res. 2011;72(3):e161-8.

16. Garcia-Bailo B, Brenner DR, Nielsen D, Lee HJ, Domanski D, Kuzyk M, Borchers $\mathrm{CH}$, Badawi A, Karmali MA, El-Sohemy A. Dietary patterns and ethnicity are associated with distinct plasma proteomic groups. Am J Clin Nutr. 2012;95(2): 352-61.

17. Bakhshani NM, Hosseinbor M, Shahraki Z, Sakhavar N. Premenstrual syndrome symptomatology among married women of fertile age based on methods of contraception (hormonal versus non-hormonal methods of contraception). Glob J Health Sci. 2014;6(2):105-11.

18. Dennerstein L, Lehert P, Keung LS, Pal SA, Choi D. A population-based survey of Asian women's experience of premenstrual symptoms. Menopause Int. 2010;16(4):139-45.

19. Goker A, Artunc-Ulkumen B, Aktenk F, Ikiz N. Premenstrual syndrome in Turkish medical students and their quality of life. J Obstet Gynaecol. 2015; 35(3):275-8.

20. Sievert LL, Bertone-Johnson E. Perimenstrual symptoms and symptoms at midlife in Puebla, Mexico. Climacteric. 2013;16(1):169-78.

21. Boyle CA, Berkowitz GS, Kelsey JL. Epidemiology of premenstrual symptoms. Am J Public Health. 1987;77(3):349-50.

22. Stout AL, Grady TA, Steege JF, Blazer DG, George LK, Melville ML. Premenstrual symptoms in black and white community samples. Am J Psychiatry. 1986; 143(11):1436-9.

23. Woods NF, Most A, Dery GK. Prevalene of perimenstrual symptoms. Am J Public Health. 1982;72(11):1257-64.

24. Lee AM, So-Kum Tang C, Chong C. A culturally sensitive study of premenstrual and menstrual symptoms among Chinese women. J Psychosom Obstet Gynaecol. 2009;30(2):105-14

25. Sadler C, Smith H, Hammond J, Bayly R, Borland S, Panay N, Crook D, Inskip H, Southampton Women's Survey Study G. Lifestyle factors, hormonal contraception, and premenstrual symptoms: the United Kingdom Southampton Women's Survey. J Women's Health (Larchmt). 2010;19(3):391-6.

26. Potter J, Bouyer J, Trussell J, Moreau C. Premenstrual syndrome prevalence and fluctuation over time: results from a French population-based survey. J Women's Health (Larchmt). 2009;18(1):31-9.

27. Joffe $H$, Cohen LS, Harlow BL. Impact of oral contraceptive pill use on premenstrual mood: predictors of improvement and deterioration. Am J Obstet Gynecol. 2003;189(6):1523-30.

28. Yonkers KA, O'Brien PM, Eriksson E. Premenstrual syndrome. Lancet. 2008; 371(9619):1200-10.

29. Kelderhouse K, Taylor JS. A review of treatment and management modalities for premenstrual dysphoric disorder. Nurs Womens Health. 2013;17(4):294-305

30. Wichianpitaya J, Taneepanichskul S. A comparative efficacy of low-dose combined oral contraceptives containing desogestrel and drospirenone in premenstrual symptoms. Obstet Gynecol Int. 2013;2013:487143.

31. Legro RS, Pauli JG, Kunselman AR, Meadows JW, Kesner JS, Zaino RJ Demers LM, Gnatuk CL, Dodson WC. Effects of continuous versus cyclical oral contraception: a randomized controlled trial. J Clin Endocrinol Metab. 2008:93(2):420-9.

32. Josse AR, Garcia-Bailo B, Fischer K, El-Sohemy A. Novel effects of hormonal contraceptive use on the plasma proteome. PLoS One. 2012;7(9):e45162

33. Bertone-Johnson ER, Ronnenberg AG, Houghton SC, Nobles C, Zagarins SE, Takashima-Uebelhoer BB, Faraj JL, Whitcomb BW. Association of inflammation markers with menstrual symptom severity and premenstrual syndrome in young women. Hum Reprod. 2014;29(9):1987-94.

34. Gold EB, Wells C, Rasor MO. The association of inflammation with premenstrual symptoms. J Women's Health (Larchmt). 2016;25(9):865-74.

35. Marvan ML, Cortes-Iniestra S. Women's beliefs about the prevalence of premenstrual syndrome and biases in recall of premenstrual changes. Health Psychol. 2001:20(4):276-80.

\section{Submit your next manuscript to BioMed Central and we will help you at every step:}

- We accept pre-submission inquiries

- Our selector tool helps you to find the most relevant journal

- We provide round the clock customer support

- Convenient online submission

- Thorough peer review

- Inclusion in PubMed and all major indexing services

- Maximum visibility for your research

Submit your manuscript at www.biomedcentral.com/submit
) Biomed Central 